\title{
O-GlcNAc elevation through activation of the hexosamine biosynthetic pathway enhances cancer cell chemoresistance
}

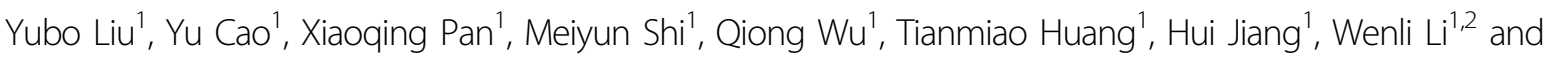 \\ Jianing Zhang ${ }^{1}$
}

\begin{abstract}
Chemoresistance has become a major obstacle to the success of cancer therapy, but the mechanisms underlying chemoresistance are not yet fully understood. O-GlcNAcylation is a post-translational modification that is regulated by the hexosamine biosynthetic pathway (HBP) and has an important role in a wide range of cellular functions. Here we assessed the role of O-GlcNAcylation in chemoresistance and investigated the underlying cellular mechanisms. The results showed that the HBP has an important role in cancer cell chemoresistance by regulating O-GlcNAcylation. An increase in the levels of O-GlcNAcylation indicates an increased resistance of cancer cells to chemotherapy. Acute treatment with doxorubicin (DOX) or camptothecin (CPT) induced O-GlcNAcylation through HBP activation. In fact, the chemotherapy agents activated the AKT/X-box-binding protein 1 (XBP1) axis and then induced the HBP. Furthermore, the observed elevation of cellular O-GlcNAcylation led to activation of survival signalling pathways and chemoresistance in cancer cells. Finally, suppression of O-GlcNAcylation reduced the resistance of both established and primary cancer cells to chemotherapy. These results provide significant novel insights regarding the important role of the HBP and O-GICNAcylation in regulating cancer chemoresistance. Thus, O-GlcNAc inhibition might offer a new strategy for improving the efficacy of chemotherapy.
\end{abstract}

\section{Introduction}

Chemotherapy is one of the standard treatment methods for many cancers and the development of chemoresistance, either intrinsic or acquired, is the most commonly encountered phenomenon that limits the success of cancer chemotherapy ${ }^{1,2}$. Understanding the mechanisms through which chemoresistance occurs has huge implications for potentiating the cancer cell-killing effect of chemotherapy. The chemoresistance of cancer cells involves complicated mechanisms, including the overexpression of multidrug efflux transporters, such as

Correspondence: Jianing Zhang (jnzhang@dlut.edu.cn)

${ }^{1}$ School of Life Science and Medicine, Dalian University of Technology, Panjin, China

${ }^{2}$ School of Life Science and Biotechnology, Dalian University of Technology, Dalian, China

Edited by A. Finazzi-Agrò
P-glycoprotein (P-gp), the activation of pro-survival pathways and ineffective induction of cell death ${ }^{2}$. However, the mechanisms modulating chemoresistance in cancers are not completely clear.

A growing body of evidence demonstrates that cancer metabolic reprogramming might influence the expression of genes to drive oncogenesis and maintain cell viability in response to stress, including drug treatment ${ }^{3,4}$. For example, the glycolytic metabolism not only alters transcription but also affects the repair of DNA damage by having an impact on the global chromatin structure in cancer cells ${ }^{5,6}$. Most malignant tissues have increased glucose uptake associated with increased rates of glycolysis and glucose transport ${ }^{7}$. Even though the majority of glucose enters glycolysis, $\sim 2-5 \%$ of glucose influx is directed toward the hexosamine biosynthetic pathway (HBP). This pathway generates UDP-GlcNAc, which is a

\section{(c) The Author(s) 2018}

(c) (i) Open Access This article is licensed under a Creative Commons Attribution 4.0 International License, which permits use, sharing, adaptation, distribution and reproduction cc) in any medium or format, as long as you give appropriate credit to the original author(s) and the source, provide a link to the Creative Commons license, and indicate if changes were made. The images or other third party material in this article are included in the article's Creative Commons license, unless indicated otherwise in a credit line to the material. If material is not included in the article's Creative Commons license and your intended use is not permitted by statutory regulation or exceeds the permitted use, you will need to obtain permission directly from the copyright holder. To view a copy of this license, visit http://creativecommons.org/licenses/by/4.0/. 


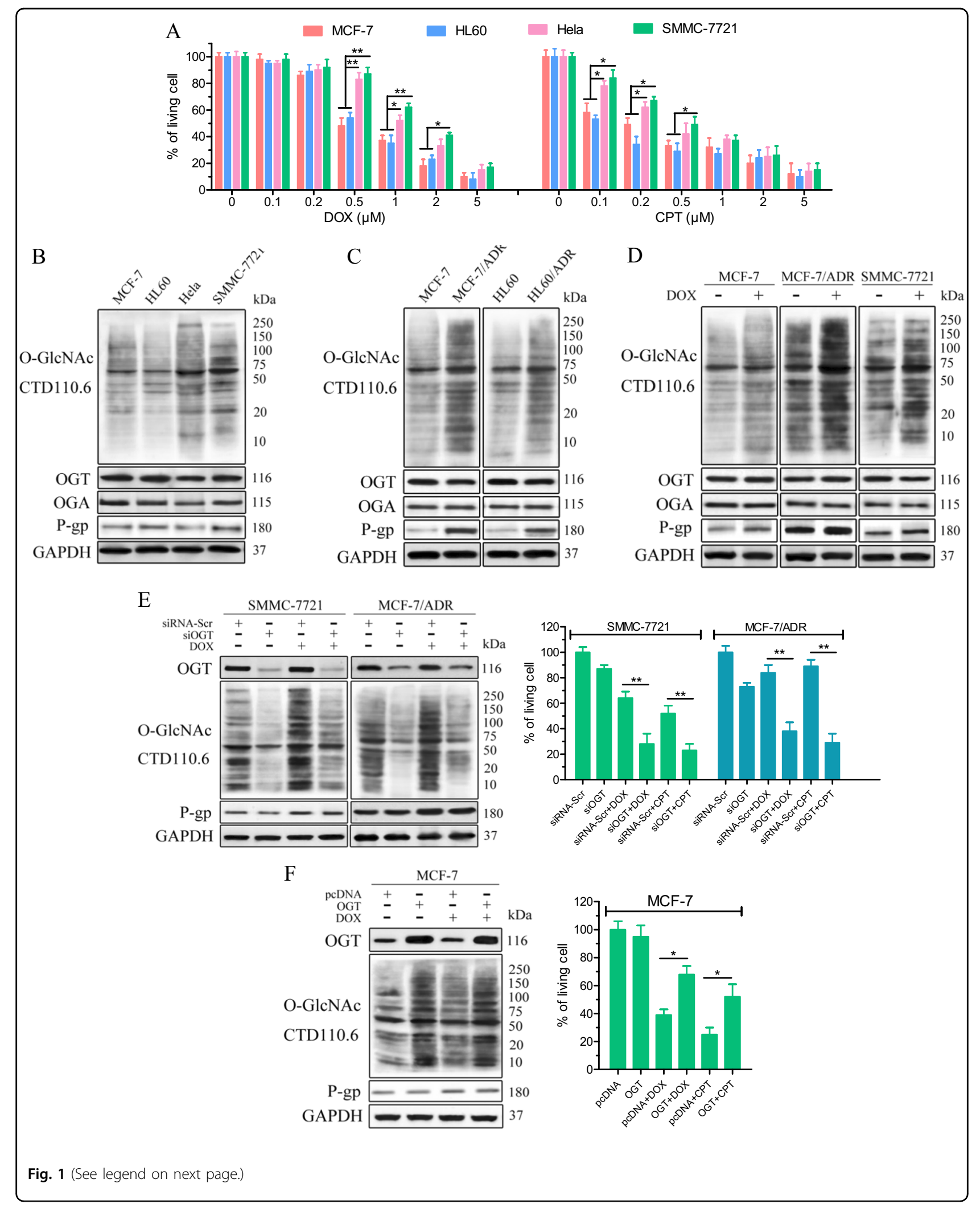


(see figure on previous page)

Fig. 1 A high level of protein O-GIcNAcylation is associated with chemoresistance in cancer cell lines. a MCF-7, HL60, Hela and SMMC-7721 cells were treated with the indicated dose of DOX or CPT for $24 \mathrm{~h}$. The cell viability was assessed through the MTS assay. $\mathbf{b}$ and $\mathbf{c}$ Cells with intrinsic and acquired chemoresistance contain elevated levels of O-GlcNAc-modified proteins compared with sensitive cells. The cellular O-GlcNAcylation and expression of indicated proteins in parental and resistant cancer cell lines were analysed by immunoblotting analysis. $\mathbf{d}$ O-GIcNAcylation is upregulated in both parental and resistant cancer cells after transient treatment with DOX. The indicated cells were treated with DOX (0.1 $\mu \mathrm{M}$ for MCF-7, $1 \mu \mathrm{M}$ for MCF-7/ADR and SMMC-7721) for $6 \mathrm{~h}$. Whole-cell lysates were obtained after treatment and analysed by immunoblotting. e The indicated cell lines were transfected with scrambled siRNA (siRNA-Scr) or OGT siRNA (siOGT) for $48 \mathrm{~h}$ and then treated with $1 \mu \mathrm{M}$ DOX or $0.5 \mu \mathrm{M}$ CPT for an additional time. The protein levels after treatment with DOX or CPT for $6 \mathrm{~h}$ were examined by immunoblotting. The cell viability after treatment with DOX or CPT for $24 \mathrm{~h}$ was assessed through an MTS assay. f MCF-7 cells were transfected with control (pcDNA) or OGT-overexpressing vector (OGT) for $48 \mathrm{~h}$ and then treated with $1 \mu \mathrm{M}$ DOX or $0.5 \mu \mathrm{M}$ CPT for an additional time. The protein levels after treatment with DOX or CPT for $6 \mathrm{~h}$ were examined by immunoblotting. The cell viability after treatment with DOX or CPT for $24 \mathrm{~h}$ was assessed through an MTS assay. The data represent the means \pm SEM, $N=3,{ }^{*} p<0.05,{ }^{* *} p<0.01$

nucleotide sugar substrate involved in multiple biological processes, including classical glycosylation and OGlcNAcylation $^{8,9}$. The available evidence suggests that alteration of the pool of activated substrates might lead to aberrant O-GlcNAcylation ${ }^{10}$. Thus, the HBP might link the altered cancer metabolism with aberrant glycosylation, providing a mechanism for how cancer cells sense and respond to a variety of cellular stress, including chemotherapy.

O-GlcNAcylation is a dynamic and reversible glycosylation of serine or threonine residues in a variety of nuclear and cytoplasmic proteins. The addition of $\mathrm{O}$ GlcNAc to proteins is catalysed by O-GlcNAc transferase (OGT) and its removal is catalysed by O-GlcNAcase (OGA). As a post-translational modification, OGlcNAcylation regulates a wide range of cellular functions. In response to numerous forms of cellular stress or injury, including DNA damage, the O-GlcNAcylation levels are dynamically elevated in both in vitro and in vivo models $^{9,11,12}$. Many O-GlcNAcylated proteins bind double-stranded DNA-dependent protein kinase or double-stranded DNA breaks, suggesting a role for OGlcNAcylation in regulating signalling pathways related to DNA damage repair and cell survival ${ }^{11,13-15}$. Together, these data indicate that HBP-induced O-GlcNAcylation might directly influence cell survival and resistance to DNA-targeting chemotherapy. However, the molecular mechanism(s) through which the HBP and OGlcNAcylation regulate thresholds in cell death and enhance cell resistance have not been identified.

In this study, we investigated the relevant role of the HBP and O-GlcNAcylation in the route leading to cancer cell resistance to chemotherapy and obtained novel mechanistic data demonstrating that chemotherapy induces flux through the HBP and then elevates cellular O-GlcNAcylation, resulting in the activation of survival-related signalling pathways and chemoresistance in cancer cells. The findings demonstrate that the combination of chemotherapy with O-GlcNAcylation inhibition bypasses chemoresistance in both established and primary cancer cells.

\section{Results}

Level of protein O-GIcNAcylation correlates with the cellular response to chemotherapy

We first investigated whether the protein $\mathrm{O}$ GlcNAcylation levels contribute to the response of cells to chemotherapy. A panel of tumour cell lines (MCF-7, HL60, Hela and SMMC-7721) were treated with $0.1-5 \mu \mathrm{M}$ doxorubicin (DOX) or camptothecin (CPT) for $24 \mathrm{~h}$. As shown in Fig. 1a, Hela and SMMC7721 cells exhibited more resistance than MCF-7 and HL60 cells. In agreement with our conjecture, the immunoblotting results showed that Hela and SMMC7721 cells contained higher levels of O-GlcNAcmodified proteins than sensitive MCF-7 and HL60 cells (Fig. 1b). However, analysis of the enzymes that govern the O-GlcNAc moiety, OGT and OGA, revealed no clear correlation between the expression levels of these proteins and the cellular response to DOX and CPT. In addition, the multidrug resistance-related P-gp levels were also not directly associated with the cellular response to chemotherapy.

We also investigated whether O-GlcNAc modification participates in the acquired resistance of chemotherapy. DOX-resistant (ADR) variants were derived from MCF-7 and HL60 cells following continuous exposure to DOX for several months (Fig. S1A). Increases in cellular OGlcNAcylation were observed in MCF-7/ADR and HL60/ ADR cells compared with their parental cell lines (Fig. 1c). Although P-gp was upregulated, the expression levels of OGT and OGA remained constant across the parental and ADR sublines.

The O-GlcNAcylation levels showed moderate increases in MCF-7 cells and significant increases in MCF-7/ ADR and SMMC-7721 cells upon acute treatment with DOX (Fig. 1d). Consistently, invisible changes in the OGT and OGA protein levels were observed in cells subjected to acute DOX. Similar results were obtained in SMMC7721 and MCF-7/ADR treated with another frontline chemotherapeutic drug 5-Fluoracil (5-FU, Fig. S1B and $\mathrm{S} 4 \mathrm{~A}$ ), indicating that the direct donor (UDP-GlcNAc) 
cellular levels of this modification was upregulated during drug stress. Further, our results showed that DOXinduced dynamic O-GlcNAcylation in resistant cells was decreased by OGT silencing (Fig. 1e). Correspondingly, OGT small interferig RNA (siRNA)-transfected cells showed increased sensitivity to DOX and CPT. In contrast, OGT overexpression in the parental MCF-7 cells reduced the levels of cell death in response to DOX (Fig. 1f). However, regardless of the OGT expression levels, P-gp can be markedly induced by DOX treatment in these cells, suggesting that O-GlcNAcylation potentiates chemotherapy resistance in a P-gp-independent manner.

In summary, these results indicate that an impact on the O-GlcNAcylation level protects cancer cells from cancer drug-induced cell death.

\section{The HBP is activated in cancer cells following exposure to chemotherapy agents}

We then measured the cellular levels of UDP-GlcNAc and activation of the HBP after drug treatment. Through liquid chromatography, we were able to distinguish UDPGlcNAc from UDP-GalNAc, and these distinct chemical species were further characterized by mass spectrum (MS) (Fig. 2a and S2). A progressive increase in the UDPGlcNAc levels was observed in the DOX-treated MCF-7/ ADR and SMMC-7721 cell extracts, and up to twofold (6 h) and fourfold (24h) higher levels of UDP-GlcNAc were observed after DOX treatment compared with the untreated MCF-7/ADR cells. Similar results were observed in SMMC-7721 cells. We then evaluated the protein levels and activities of fructose-6-phosphate amidotransferase (GFAT), the key rate-limiting enzymes of the $\mathrm{HBP}^{16}$. The GFAT levels (messenger RNA and protein) were higher in SMMC-7721 and MCF-7/ADR cells than in MCF-7 cells (Fig. 2b). Further GFAT accumulation and increased GFAT activity were observed during DOX treatment in these cells (Fig. 2b,c). We also investigated the protein levels and activities of phosphofructokinase (PFK) and glucose 6-phosphate dehydrogenase (G6PD), which control the glucose metabolism through the glycolytic and pentose phosphate pathways, respectively ${ }^{17}$. No significant changes were observed after DOX treatment. Confirming a role for the HBP in drug resistance, GFAT inhibition with azaserine $(\mathrm{AZA})^{18}$ significantly induced SMMC-7721 and MCF-7/ADR cell death under DOX treatment without affecting the expression of OGT and P-gp (Fig. 2d). In addition, the levels of O-GlcNAcylated proteins were reduced by GFAT inhibition and correspondingly similar results were obtained with GFAT siRNA (Fig. 2e). These results indicate upregulation of flux through the HBP during drug stress, revealing an uncharacterized regulation of the HBP in chemotherapy resistance.

\section{Activation of the AKT/XBP1 axis by chemotherapy regulates flux through the HBP}

AKT has been implicated in regulation of the expression and activity of XBP1, which is the transcription factor of GFAT $^{19,20}$. This study found that the phosphorylation of AKT on serine 473, a phosphatidylinositol-3-kinase (PI3K)-dependent target, was significantly higher in DOX-treated MCF-7/ADR cells than in MCF-7 cells, concomitant with the more efficient induction of X-boxbinding protein 1 (XBP1) and GFAT (Fig. 3a). XBP1 splicing was also checked by reverse-transcriptase PCR (RTPCR) (Fig.S3). Similarly, in SMMC-7721 cells, the AKT/ XBP1 axis was also activated upon DOX treatment. AKT inhibitor MK2206 ${ }^{21}$ abrogated the induction of XBP1 and GFAT by DOX treatment. In fact, a slight XBP1 induction was observed, indicating that other signalling pathways have roles in drug-induced HBP activation. Consistent results were observed in the cells treated with 5-FU (Fig. S4A). Furthermore, the induction of UDP-GlcNAc by DOX treatment in SMMC-7721 and MCF-7/ADR cells was also significantly attenuated by MK2206 (Fig. 3b), confirming that the inhibition of AKT compromises the potency of $\mathrm{HBP}$ activation.

SMMC-7721 and MCF-7/ADR cells were further treated with siRNA targeting XBP1. As expected, XBP1 acted upstream of GFAT and, as such, the downregulation of XBP1 reduced GFAT expression and O-GlcNAc modification of proteins in DOX-resistant cells (Fig. 3c). We also noticed an additional $30-40 \%$ cell death, which increased with the duration of the XBP1 siRNA treatment, compared with DOX treatment alone. To explore the role of unfolded protein response (UPR ${ }^{20}$ in drug-induced HBP activation, the classic UPR inhibitor versipelostatin (VST) was used ${ }^{22}$ and the results showed that VST suppressed the accumulation of endogenous GRP78 (a commonly used indicator of UPR activation) in SMMC7721 and MCF-7/ADR cells under DOX treatment. However, the downregulation of GRP78 expression partially inhibited XBP1 and downstream GFAT, indicating a UPR-independent mechanism for the regulation of XBP1 (Fig. 3d). Similar results were obtained with the knockdown of GRP78 using siRNA (Fig. S4B). Further analysis showed that pAKT (S473) was still inducible upon DOX treatment regardless of whether GRP78 was inhibited by VST or GRP78 siRNA. The combination of VST and MK2206 almost completely inhibited the induction of XBP1 and GFAT. These data provide compelling evidence that AKT activation by chemotherapy increases the flux through the HBP in a UPR-independent manner.

Taken together, these results suggest that activation of the HBP by chemotherapy could be a common mechanism through which cancer cells antagonize cell death and this mechanism is at least partially mediated through the AKT/XBP1 axis. 


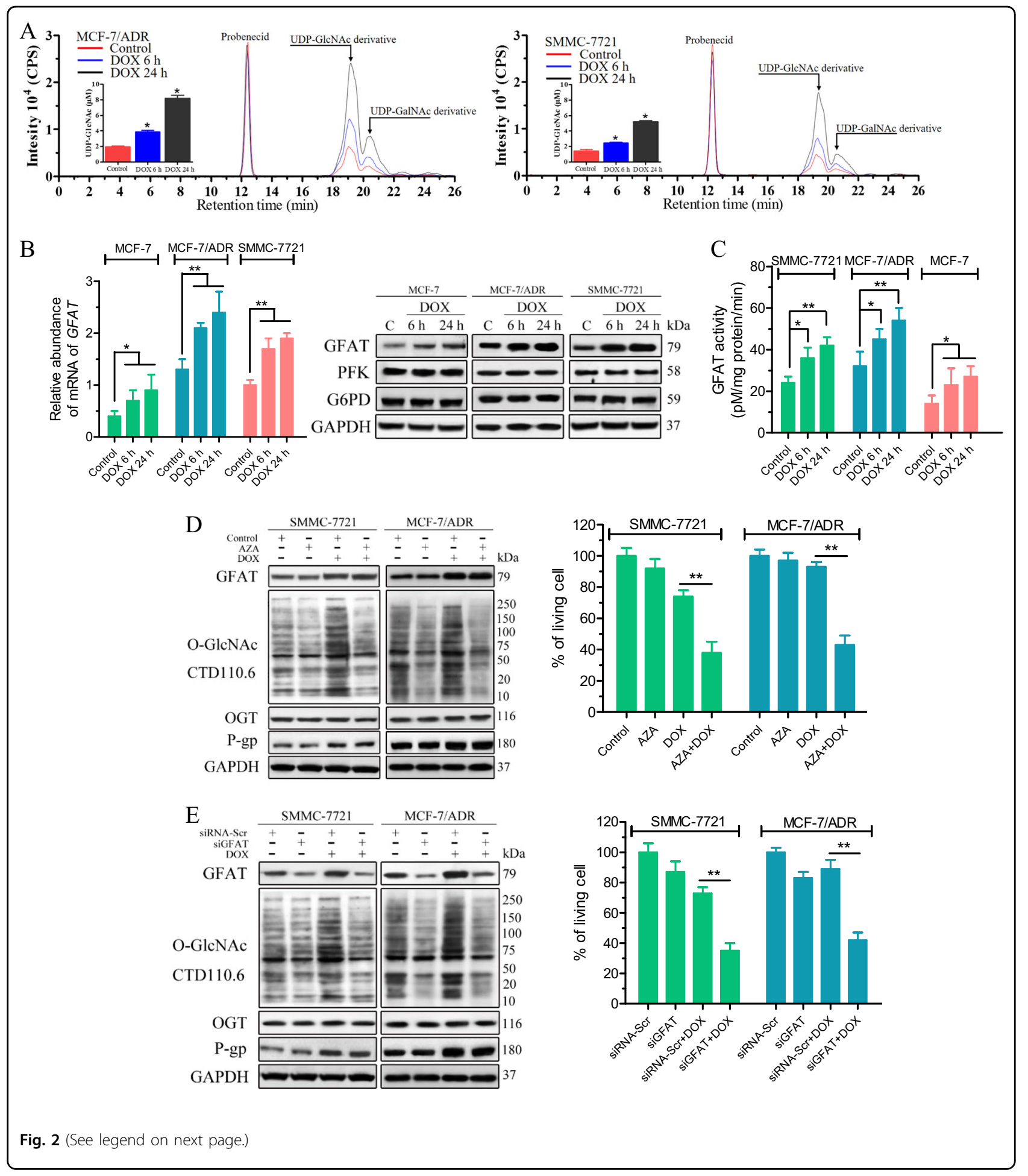

Elevated O-GIcNAcylation of prosurvival proteins suppress cell death in response to chemotherapy

We next investigated the downstream targets of $\mathrm{O}$ GlcNAcylation. A previous study found that the cleavage of apoptotic caspases could be blocked by O-
GlcNAcylation $^{23}$. In our study, the major intrinsic apoptotic caspases, namely caspase-3 and 9, were also $\mathrm{O}$ GlcNAcylated in the parental and resistant cells. In MCF7 and its resistant subline, O-GlcNAcylated caspase- 3 was absent, because these are caspase-3-null cell lines. 
(see figure on previous page)

Fig. 2 Flux through the HBP in cancer cells is up-regulated in response to drug stress. a DOX induces increases in the UDP-GICNAC levels. UDPGICNAc in the cell lysates were derivatizated with trimethylsilyldiazomethane. Chromatograms of polar metabolites in MCF-7/ADR and SMMC-7721 cell extracts from control (red line), and $1 \mu \mathrm{M}$ DOX-treated cells for $6 \mathrm{~h}$ (blue line) and $24 \mathrm{~h}$ (black line) show regions corresponding to the UDPGlcNAc derivative, UDP-GaINAc derivative and probenecid retention times. Quantitative analyses are shown as means \pm SEM, deviation of three independent experiments. $P$-values were calculated using one-way ANOVA and the appropriate post test. ${ }^{*} P<0.05$. $\mathbf{b}$ The GFAT transcriptional and protein levels are upregulated in parental and resistant cell lines after transient treatment with DOX. MCF-7, MCF-7/ADR and SMMC-7721 cells were treated with DOX (0.1 $\mu \mathrm{M}$ for MCF-7, $1 \mu \mathrm{M}$ for MCF-7/ADR and SMMC-7721) for indicated time and the GFAT transcript level was analysed by quantitative PCR. DMSO was used as a control. Whole-cell lysates were analysed by immunoblotting. c MCF-7, MCF-7/ADR and SMMC-7721 cells were treated with DOX (0.1 $\mu \mathrm{M}$ for MCF-7, $1 \mu \mathrm{M}$ for MCF-7/ADR and SMMC-7721) for the indicated time and cell lysates were used for the analysis of GFAT activity. d SMMC-7721 and MCF-7/ADR cells were treated with $1 \mu \mathrm{M}$ DOX alone or together with $5 \mu \mathrm{M}$ AZA for 6 and $24 \mathrm{~h}$. The protein levels after treatment with DOX or AZA for $6 \mathrm{~h}$ were examined by immunoblotting. DMSO was used as a control. The cell viability after treatment with DOX or AZA for $24 \mathrm{~h}$ was assessed through an MTS assay. e The indicated cell lines were transfected with scrambled siRNA (siRNA-Scr) or GFAT siRNA (siGFAT) for $48 \mathrm{~h}$ and then treated with $1 \mu \mathrm{M}$ DOX. The protein levels after treatment with DOX for $6 \mathrm{~h}$ were examined by immunoblotting. The cell viability after treatment with DOX for $24 \mathrm{~h}$ was assessed through an MTS assay. The data represent the means $\pm \mathrm{SEM}, N=3,{ }^{*} p<0.05,{ }^{* *} p<0.01$

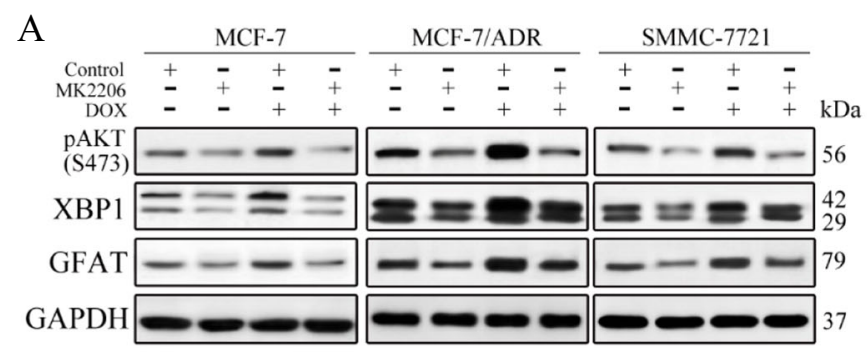

$\mathrm{B}$
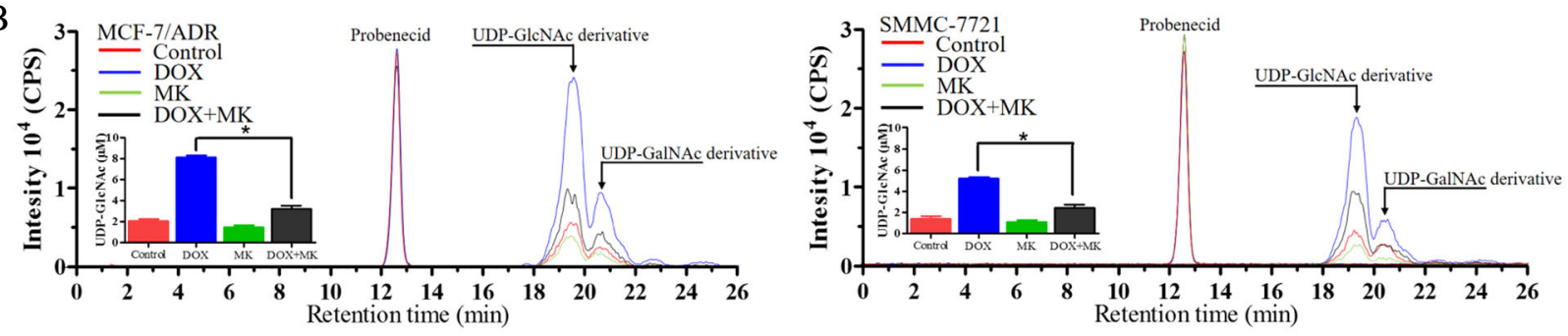

$\mathrm{C}$

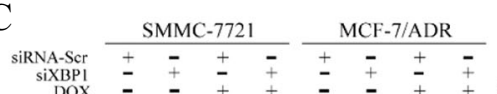
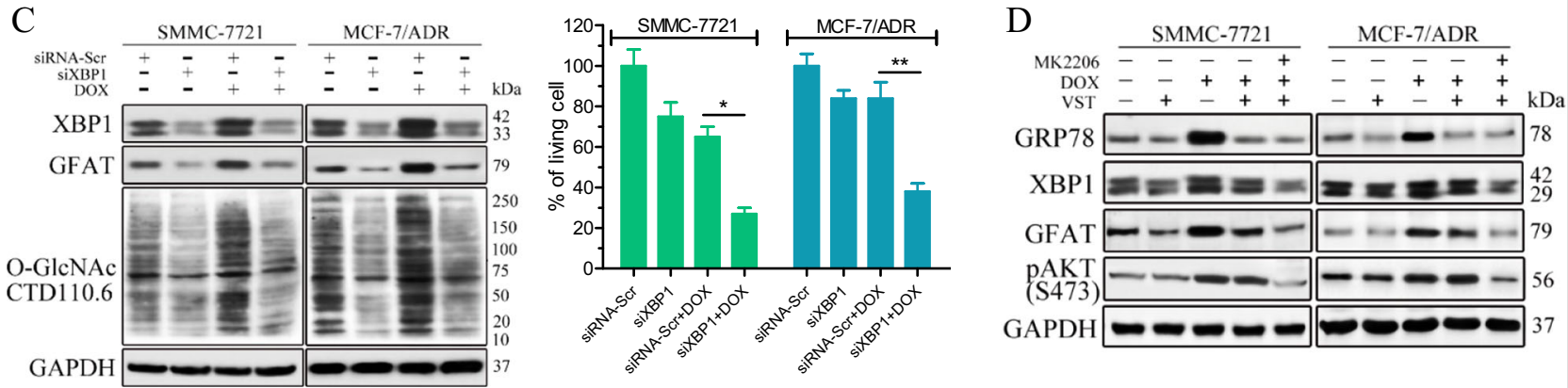

Fig. 3 Chemotherapy-induced HBP activation is regulated by the AKT/XBP-1 axis. a AKT has a role in activating XBP1 and GFAT. The indicated cells were treated with DOX (0.1 $\mu \mathrm{M}$ for MCF-7, $1 \mu \mathrm{M}$ for MCF-7/ADR and SMMC-7721) alone or together with $1 \mu \mathrm{M}$ MK2206 for $6 \mathrm{~h}$. DMSO was used as a control. The protein levels were examined by immunoblotting. blnduction of UDP-GlcNAc by DOX is attenuated by AKT inhibition. Chromatograms of polar metabolites in MCF-7/ADR and SMMC-7721 cell extracts from control cells (red line), cells treated with $1 \mu M$ DOX (blue line) or 1 MM MK2206 (green line) alone and cells treated with DOX plus MK2206 (black line) for $24 \mathrm{~h}$ show the regions corresponding to the UDP-GICNAc derivative, UDP-GalNAc derivative and probenecid retention times. c XBP1 acts upstream of GFAT. The indicated cell lines were transfected with scrambled siRNA (siRNA-Scr) or XBP1 siRNA (siXBP1) for $48 \mathrm{~h}$ and then treated with $1 \mu \mathrm{M}$ DOX. The protein levels after treatment with DOX for $6 \mathrm{~h}$ were examined by immunoblotting. The cell viability after treatment with DOX for $24 \mathrm{~h}$ was assessed through an MTS assay. $\mathbf{d}$ AKT/XBP1 axis regulates GFAT expression in a UPR-independent manner. The indicated cells were treated with $1 \mu \mathrm{M}$ DOX alone or together with $3 \mu \mathrm{M}$ VST for $6 \mathrm{~h}$. DMSO was used as a control. The protein levels were examined by immunoblotting. The data represent the means $\pm \operatorname{SEM}, N=3,{ }^{*} p<0.05,{ }^{* *} p<0.01$ 


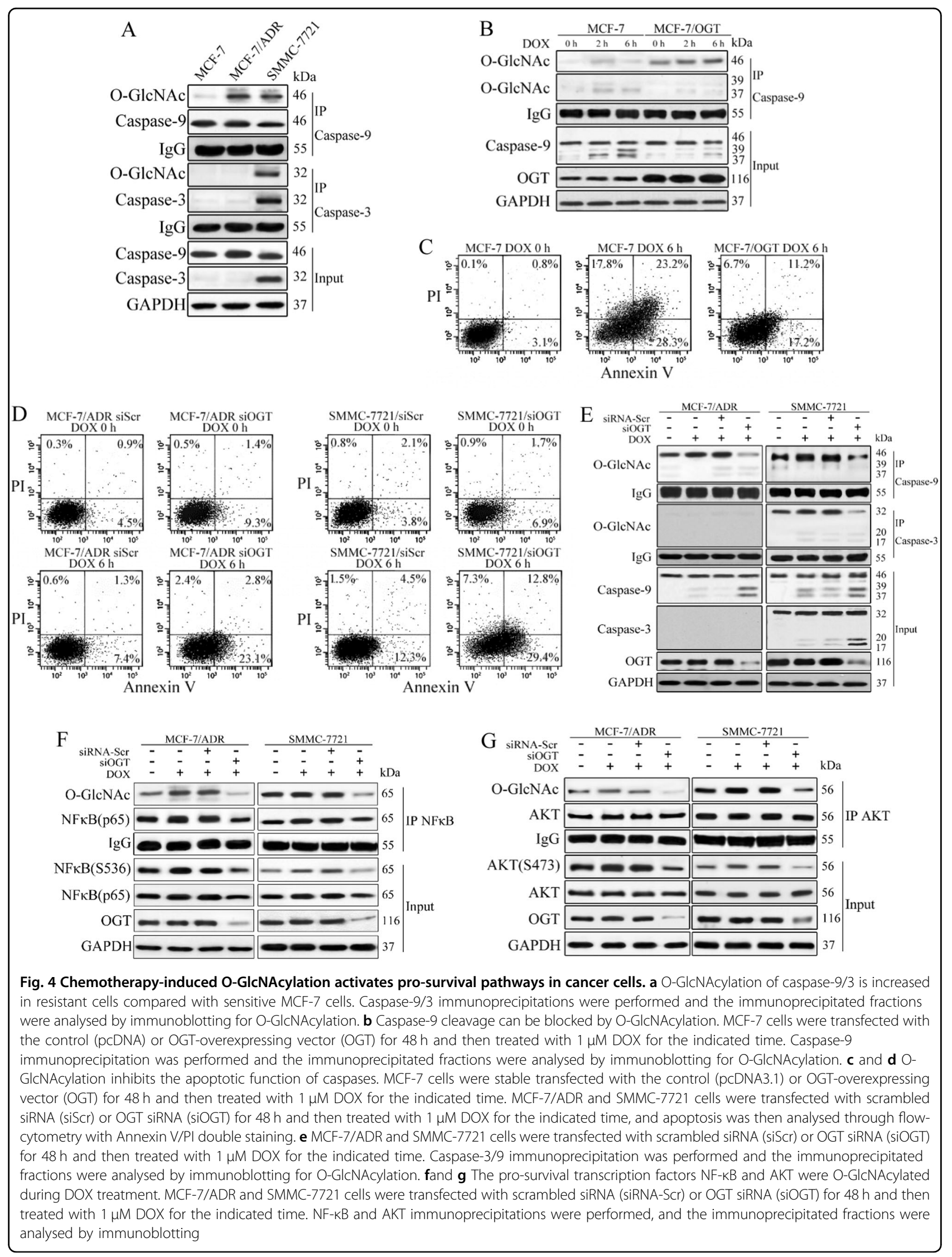



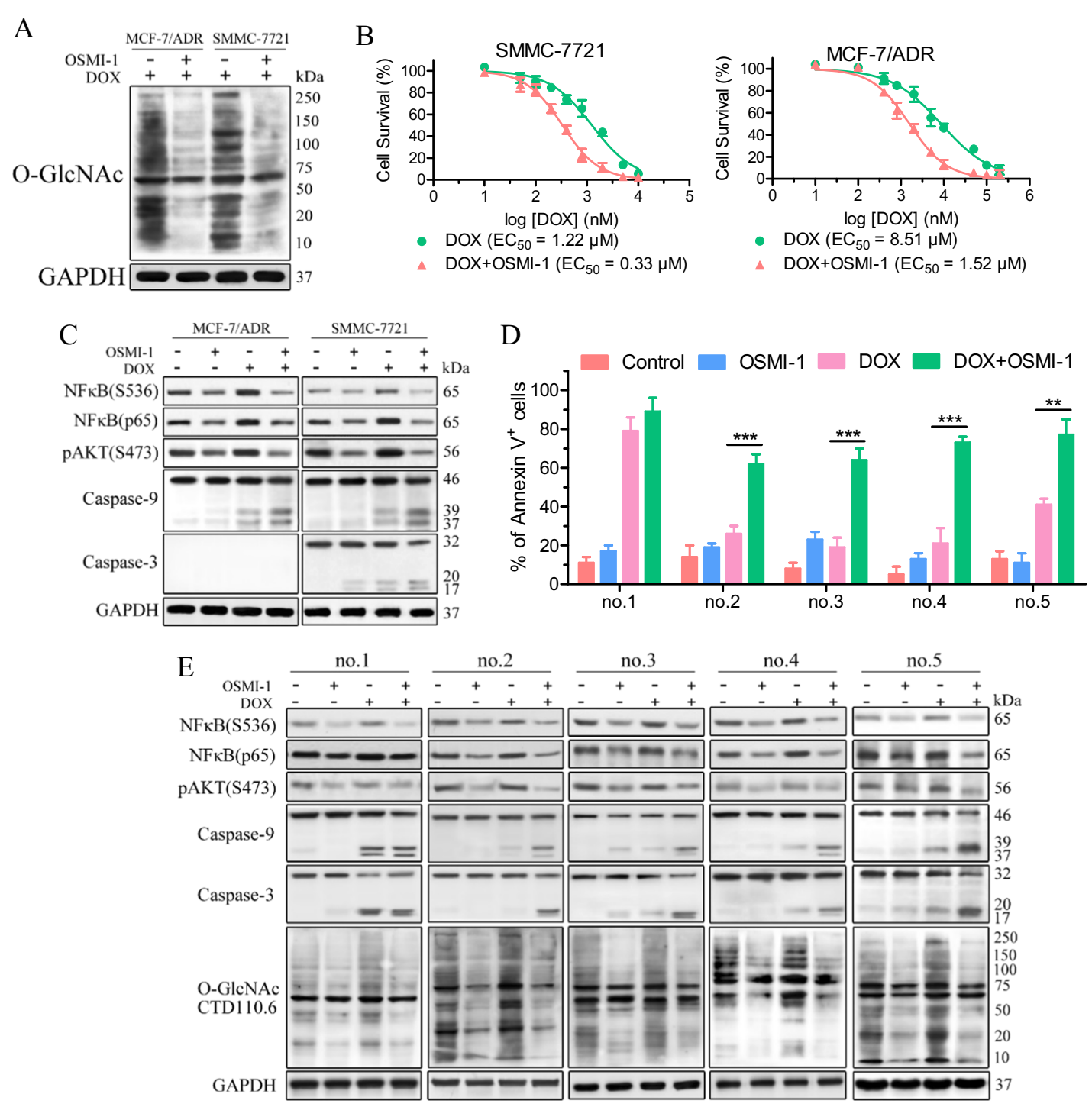

Fig. 5 Inhibition of O-GIcNAc sensitizes resistant cancer cells to chemotherapy. a SMMC-7721 and MCF-7/ADR cells were treated with $1 \mu M$ DOX alone or together with $20 \mu \mathrm{M}$ OSMI-1 for $6 \mathrm{~h}$. The protein levels were examined by immunoblotting. DMSO was used as a control. b SMMC-7721 and MCF-7/ADR cells were treated with increasing doses of DOX alone or together with $20 \mu \mathrm{M}$ OSMI- 1 for $24 \mathrm{~h}$ and the cell viability was then assessed though an MTS assay. The $\mathrm{EC}_{50}$ values were calculated. c SMMC-7721 and MCF-7/ADR cells were treated with $1 \mu \mathrm{M}$ DOX alone or together with $20 \mu \mathrm{M}$ OSMI-1 for $6 \mathrm{~h}$. The protein levels were examined by immunoblotting. DMSO was used as a control. $\mathbf{d}$ and e Primary AML cells were treated with $5 \mu \mathrm{M}$ DOX alone or together with $20 \mu \mathrm{M}$ OSMI-1 for $24 \mathrm{~h}$. The cells were then stained with Annexin $V$ for flow cytometry analysis. The protein levels were examined by immunoblotting and DMSO was used as a control. ${ }^{* *} p<0.01,{ }^{* * *} p<0.001$

Notably, the O-GlcNAcylation of these caspases was increased in resistant SMMC-7721 and MCF-7/ADR cells compared with sensitive MCF-7 cells (Fig. 4a). Treatment of MCF-7 cells with DOX resulted in continuous caspase9 activation with rare O-GlcNAcylation accumulation, whereas OGT overexpression decreased the activation rate and upregulated O-GlcNAcylated caspase-9 (Fig. 4b). Consistently, through Annexin V-fluorescein isothiocyanate (FITC)/propidium iodide (PI) staining, we found that the apoptosis rates of OGT-overexpressing cells were significantly decreased compared with the control cells in the presence of DOX (Fig. 4c). In resistant cells, DOX treatment led to further accumulation of OGlcNAcylated caspase-9 (and caspase-3 in SMMC-7721 cells) and this effect was accompanied by slight caspase cleavage. In contrast, OGT silencing in the presence of DOX enhanced cell apoptosis and activation of caspase-9 and 3, indicating that the apoptotic function of caspases can be inhibited by chemotherapy-induced O-GlcNAcylation (Fig. 4d,e).

In addition, the pro-survival transcription factors nuclear factor- $\kappa \mathrm{B}(\mathrm{NF}-\kappa \mathrm{B})$ and $\mathrm{AKT}$ were also O- 
GlcNAcylated during DOX treatment in resistant cells. OGT downregulation in SMMC-7721 and MCF-7/ADR cells decreased the NF- $\mathrm{kB}$ protein level and phosphorylation at Ser536, which is critical for NF- $\kappa B$ nuclear translocation and activation (Fig. 4f). Moreover, OGlcNAcylation of AKT enhanced AKT phosphorylation at Ser473, indicating that AKT and the HBP are activated by a positive feedback mechanism involving OGlcNAcylation (Fig. 4g). Based on the above results, we can conclude that chemotherapy-induced O-GlcNAcylation activates pro-survival pathways and strengthens the cell-death threshold of chemotherapy drugs.

\section{Combination of O-GIcNAc suppression with chemotherapy alleviates drug resistance in both established and primary cancer cells}

Blockage of the HBP or downstream O-GlcNAcylation disrupted pro-survival pathways, suggesting that $\mathrm{O}$ GlcNAc suppression is promising in overcoming the resistance of cancer cells to chemotherapy. A western blot analysis showed that DOX-induced cellular OGlcNAcylation was significantly decreased in SMMC7721 and MCF-7/ADR cells by treatment with the OGT inhibitor OSMI-1 ${ }^{24}$ (Fig. 5a). DOX alone induced SMMC7721 and MCF-7/ADR cell death with half-maximal effective concentrations $\left(\mathrm{EC}_{50}\right)$ of $1.22 \mu \mathrm{M}$ and $8.51 \mu \mathrm{M}$, respectively (Fig. 5b), whereas no substantial cell killing was observed with OSMI-1 alone $\left(\mathrm{EC}_{50}>100 \mu \mathrm{M}\right.$, Fig. S5). With the combination of DOX and OSMI-1 (20 $\mu \mathrm{M})$, the $\mathrm{EC}_{50}$ values for MCF-7/ADR cell death decreased to $1.52 \mu \mathrm{M}$ (Fig. 5b). This combination of OSMI-1 with DOX also induced a four-fold increase in the efficacy of SMMC-7721 cell killing $\left(\mathrm{DOX} \mathrm{EC}_{50}=0.33\right.$ $\mu \mathrm{M})$, which is comparable to the $\mathrm{EC}_{50}(0.34 \mu \mathrm{M})$ for the DOX-sensitive MCF-7 cell line (Fig. S6). Similar potentiation of OSMI-1 combined with CPT or 5-FU was also observed in SMMC-7721 and MCF-7/ADR cells (Fig. S7). Furthermore, $24 \mathrm{~h}$ treatment of these cells with the combination of DOX and OSMI-1 resulted in a significant decrease of activated NF- $\mathrm{kB}$ and AKT compared with DOX alone. An increase in the level of cleaved caspase-9/ 3 was also observed in the cells after combinatorial treatment (Fig. 5c). These observations suggest that the inhibition of O-GlcNAc sensitizes resistant cancer cell lines to chemotherapy.

Furthermore, five primary acute myeloid leukemia (AML) patient samples (Table S1) were examined for OSMI-1-induced sensitization to DOX (Fig. 5d). DOX treatment $(5 \mu \mathrm{M})$ led to significant cell death in a newly diagnosed untreated patient sample (no. 1). In contrast, 5 $\mu \mathrm{M}$ DOX alone mildly reduced the viability of refractory $(n=3)$ and relapsed $(n=1)$ primary cells (no. 2-5), whereas OSMI-1 hardly affected the viability of these samples. Notably, significant enhancement of OSMI-1 activity by treatment combinations with DOX was observed in these samples (no. 2-5). Cellular OGlcNAcylation upregulation was induced by DOX alone in these samples (no. 1-5), and this upregulation of OGlcNAcylation was reduced by the combination of DOX with OSMI-1. A decrease in the levels of phosphorylated NF- $K B$ and AKT accompanied with an increase in the levels of activated caspase-9/3 was observed after the DOX/OSMI-1 combination treatment (Fig. 5e). All these results agree with those observed in established cell lines and support a cooperative mechanism between chemotherapy and O-GlcNAc inhibition.

\section{Discussion}

Chemoresistance is the most significant reason for the failure of cancer chemotherapeutics and is crucial to cancer metastasis and recovery $^{1}$, but the interplay between cancer metabolic reprogramming and resistance following chemotherapy is not fully understood. Here we report that the HBP has an important role in the cellular metabolic response to chemotherapy by regulating $\mathrm{O}$ GlcNAcylation, and that this effect has important implications in drug resistance. First, we discovered that drug stress activates the HBP via the AKT/XBP1 axis. Second, $\mathrm{O}-$ GlcNAcylation is dynamically induced by the HBP and is required for cell survival and chemoresistance in cancer cells upon drug treatment. This post-translational modification contributes to the inhibition of apoptosis by blocking caspase cleavage and the activation of prosurvival transcription factors, such as NF- $\mathrm{kB}$ and AKT, in response to chemotherapy. Furthermore, suppression of the HBP or O-GlcNAcylation reduces cancer cell resistance to chemotherapy. Our studies therefore reveal that O-GlcNAcylation is an important regulator of cell-death thresholds to chemotherapy that coordinates the HBP with chemoresistance.

Among the many hallmarks of cancer, altered metabolism has returned to the forefront as a potential therapeutic target. A neglected but integral branch of glucose metabolism is the HBP, which produces the sugar substrate of O-GlcNAcylation ${ }^{25}$. In this study, cells with intrinsic and acquired resistance were characterized by higher levels of O-GlcNAcylation than sensitive and parental cells, whereas no correlation between cell sensitivities and OGT or OGA expression was found. In addition, O-GlcNAcylated proteins were dynamically increased upon acute treatment with DOX, suggesting that abnormalities in O-GlcNAcylation participates in chemoresistance. The enzyme responsible for this conjugation, OGT, is largely dependent on the cellular free UDP-GlcNAc levels for enzymatic activity. OGT expression levels did not change during drug stress, indicating that the synthesis of UDP-GlcNAc through the HBP is pivotal in regulating O-GlcNAcylation. Significant 
increases in the GFAT protein levels and activity were detected during DOX treatment, indicating that the increased metabolite levels can potentially enter the HBP. The results showed significant increases in UDP-GlcNAc and cellular O-GlcNAcylation, supporting our hypothesis that the HBP is activated during drug stress. Although UDP-GlcNAc serves as a donor substrate in multiple glycosylation reactions, the regulation of cellular $\mathrm{O}-$ GlcNAcylation by OGT knockdown and overexpression significantly sensitized the resistant cells to DOX, suggesting that O-GlcNAcylation has a key role in chemoresistance.

Multiple lines of evidence suggest that OGlcNAcylation is increased in various cellular stresses, and that this increase might be protective ${ }^{26,27}$. Our work extends the function of O-GlcNAcylation in modulating cell resistance to chemotherapy. Apoptosis-related caspase-9/3 were stimulated by drug-induced O-GlcNAcylation. This modification blocks the cleavage/activation of caspase-9/3 and serves as a potential anti-apoptotic mechanism. In addition, the O-GlcNAcylation of prosurvival transcription factors (NF- $\mathrm{KB}$ and $\mathrm{AKT}$ ) promotes their phosphorylation, which, in turn, enhances their pro-survival function. AKT activation further forms a positive feedback loop that strengthens the flux through the HBP and regulates genotoxic resistance in cancer cells. These O-GlcNAcylation-triggered prosurvival factors strengthen the thresholds that need to be overcome prior to the initiation of cell death pathways. However, our results do not exclude the possibility that other pro-survival processes, such as autophagy and mitochondrial dysfunction, could participate, together with HBP-induced O-GlcNAcylation, in determining the fate of cancer cells in response to chemotherapy treatment. Given the multiple targets of O-GlcNAcylation in epigenetic regulation, obtaining a more in-depth understanding of the precise roles of this modification in chemoresistance and cell survival requires further investigation.

Increased activity of the PI3K/Akt pathway was previously shown to participate in resistance to various chemotherapeutic agents $^{28}$. We observed that DOX treatment significantly increased AKT phosphorylation in SMMC-7721 and MCF-7/ADR cells. Pharmacological inhibition of AKT using MK2206 significant reduced the XBP1 expression level and restored the sensitivity of SMMC-7721 and MCF-7/ADR cells to DOX. A previous study indicated that the transcription factor XBP1 can directly promote the transcription of $\mathrm{GFAT}^{20}$. Indeed, XBP1 deficiency decreased GFAT expression in both SMMC-7721 and MCF-7/ADR cells in response to DOX treatment. These data uncover a mechanistic axis that directly links the HBP, O-GlcNAcylation, and chemoresistance under drug stress.
The therapeutic efficacy of first-line anticancer drugs, such as DOX, CPT and 5-FU, is limited by the resistance developed during the treatment. We demonstrated that the OGT inhibitor OSMI-1 effectively sensitized DOXresistant SMMC-7721 and MCF-7/ADR cells to DOX and 5-FU. For the uracil analogue 5-FU, although it is an analogue of uracil, 5-FU may not affect the UDP-GlcNAc synthesis in this study. The inhibition of OGlcNAcylation was also proven to serve as a useful strategy for DOX resistance in primary refractory or relapsed AML cells, suggesting that the combination of an O-GlcNAcylation inhibitor with chemotherapy could be a novel strategy in cancer therapy. However, although pharmacological targeting of OGT is possible in vitro, important work is still required for in vivo application.

Taken together, our results provide evidence showing that activation of O-GlcNAcylation by increasing flux through the HBP impacts cell survival and resistance during chemotherapy (Fig. 6). We propose that this is an important physiological response of cancer cells stimulated with chemotherapy. By promoting cell survival, HBP-induced O-GlcNAcylation might have an important role in determining cell fate and maintaining cellular immortality following drug treatment.

\section{Materials and methods \\ Cell culture and reagents}

MCF-7, HL60, Hela and SMMC-7721 cells were obtained from Type Culture Collection of the Chinese Academy of Sciences (Shanghai, China) and were used

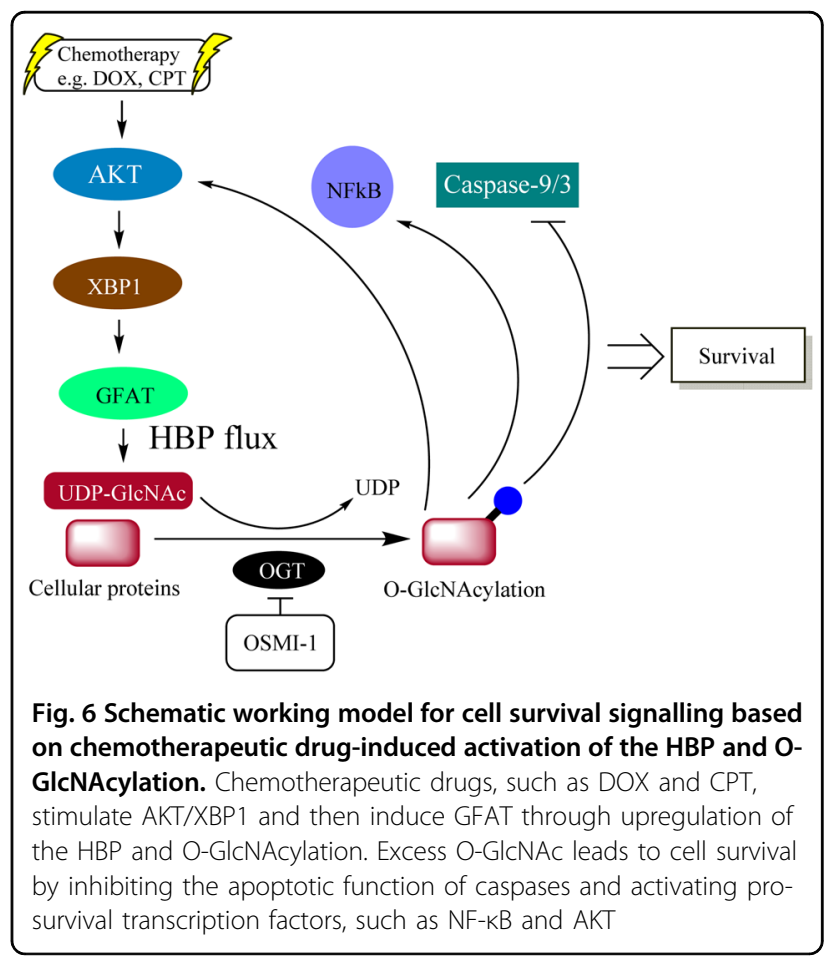


within 6 months from resuscitation. All the cells were cultured in 90\% RPMI-1640 (Gibco, USA) supplemented with $1 \%$ penicillin/streptomycin antibiotics (Gibco) and $10 \%$ fetal bovine serum (Gibco). DOX (Sigma, St Louis, $\mathrm{MO}$, USA) was added to cell cultures in stepwise increasing concentrations from 0.1 to $10 \mu \mathrm{M}$ for 4 months to develop an ADR subline, namely HL60/ADR and MCF7/ADR, correspondingly. To maintain the ADR phenotype, the complete medium of the resistant cell clones were supplemented with $2 \mu \mathrm{M}$ DOX. ADR cells were maintained in complete medium without adriamycin for 1 week and cells with $>90 \%$ viability before subsequent treatments.

Human OGT complementary DNA was cloned in pcDNA3.1 plasmid. AZA, VST, MK2206 and CPT were purchased from Sigma. Lipofectamine 2000, Annexin VFITC/PI and G418 were from Invitrogen (Grand Island, NY, USA).

\section{Isolation and culture of AML primary cells}

Cells from five patients (refractory $(n=3)$, relapsed $(n$ $=1$ ) and newly diagnosed untreated $(n=1)$ ) diagnosed with AML (except M3) according to the World Health Organization classification were studied. Refractory patients failed to achieve remission following at least two induction chemotherapy. Relapsed patient recurred after complete remission ( $<5 \%$ marrow blasts) and resisted to the re-induction protocol. Tumour cells were obtained from bone marrow and the characteristics of these patients are listed in Supplementary Table S1. In all cases, informed consent was obtained in accordance with the guidelines and the approval of the Second Affiliated Hospital of Dalian Medical University (Dalian, Liaoning, China) and the Declaration of Helsinki.

Mononuclear cells from peripheral blood samples were isolated by Ficoll-Hypaque sedimentation (Sigma Chemical Co., St Louis, MO, USA). Contaminating red cells were lysed in $0.8 \%$ ammonium chloride solution for $10 \mathrm{~min}$. The cells were either used directly or cryopreserved in liquid nitrogen in the presence of $10 \%$ dimethyl sulfoxide and $60 \%$ fetal calf serum (Gibco BRL, Grand Island, NY, USA). Cells from AML patients were cultured in RPMI 1640 culture medium supplemented with $10 \%$ fetal bovine serum and $1 \%$ penicillin/streptomycin antibiotics.

\section{Quantitative RT-PCR}

Total RNA was isolated using the Trizol method (Invitrogen). A total of $5 \mu \mathrm{g}$ of RNA were reversetranscribed and amplified using One Step SYBR PrimeScript PLUS RT-PCR Kit (TaKaRa, Dalian, China) and the Thermal Cycler Dice instrument (TaKaRa) according to the manufacturer's instructions. RT-PCR primers for GADPH and GFAT are listed as follows: GADPH sense:
5'- TGGTGAAGCAGGCATCTGAG-3', antisense: 5' CTCCTGCGACTTCAACAGCA-3'; GFAT sense: 5'AACTACCATGTTCCTCGAACGA-3', antisense: $5^{\prime}$ CTCCATCAAATCCCACACCAG-3'. Results were normalized to GAPDH.

\section{Immunoblotting and immunoprecipitation}

The cell lysing, western blotting and immunoprecipitation were performed as previously described ${ }^{29}$. The following antibodies were used: OGT, OGA, GAPDH, Pgp, AKT (Rabbit), XBP1, GFAT, PFK and G6PD were from Abcam (Hong Kong, China). pAKT (S473), AKT (mouse), GRP78, Caspase-9 and Caspase-3 were from Cell Signaling Technology (MA, USA); O-GlcNAcylation antibody (CTD110.6) was from BioLegend (MA, USA). Chemiluminescent detection was performed using ECL kit (GE Healthcare, USA).

\section{Cell viability and apoptosis assays}

Cells were treated for different times in 96-well culture plates in $200 \mu \mathrm{L}$ of culture medium. Each concentration was tested in duplicate at least thrice separately. The viable cells were determined using the CellTiter 96 AQueous non-radioactive cell proliferation MTS assay (Promega) and the inhibition rates were analysed using GraphPad 5. Cell apoptosis was assessed by Annexin-V/PI staining using a FACSCalibur flow cytometry system.

\section{Gene silencing and stable transfection}

Gene silencing was achieved though transfection with siRNA using the Lipofectamine 2000 transfection reagent following the manufacturer's instructions. OGT siRNA (sc-40780), GFAT siRNA (sc-6068), XBP1 siRNA (sc38627), GRP78 siRNA (sc-29338) and scrambled siRNA (sc-37007) were obtained from Santa Cruz Biotechnology (CA, USA).

Transfection of the MCF-7 cell line was performed with Lipofectamine according to the manufacturer's instructions. Under our conditions, $30-40 \%$ of cells are routinely transfected. The stably transfected cells were then selected by the addition of $800 \mu \mathrm{g} / \mathrm{mL}$ geneticin (G418), which was purchased from Invitrogen, to the medium. After 3 weeks, the selected stably transfected cells were further cultured with G418 at a concentration of $400 \mu \mathrm{g} / \mathrm{mL}$ and OGT-overexpressing clones were selected for other experiments.

\section{Statistical analysis}

All data are presented as the mean $\pm \mathrm{SEM}, N=3$. Data groups were compared by two-tailed Student's $t$-test using the GraphPad Software. Differences between groups were considered statistically significant if $p<0.05$. The statistical significance is denoted by asterisks $\left({ }^{*} p<0.05\right.$; ${ }^{* * *} p<$ $0.01 ; * * * 0.001)$. 


\section{Acknowledgements}

We gratefully acknowledge support from Natural Science Foundation of China (21502015, 31570802), the Fundamental Research Funds for the Central Universities (DUT17JC21). We also thank Professor David J. Vocadlo (Simon Fraser University, Canada) for providing plasmids.

\section{Author details}

'School of Life Science and Medicine, Dalian University of Technology, Panjin, China. ${ }^{2}$ School of Life Science and Biotechnology, Dalian University of Technology, Dalian, China

\section{Author contributions}

JZ conceived and designed the study. All authors designed and performed individual experiments and analysed the data. JZ and $\mathrm{YL}$ drafted the manuscript, while all authors reviewed and approved the final version of the manuscript.

\section{Conflict of interest}

The authors declare that they have no conflict of interest.

\section{Publisher's note}

Springer Nature remains neutral with regard to jurisdictional claims in published maps and institutional affiliations.

Supplementary Information accompanies this paper at (https://doi.org/ 10.1038/s41419-018-0522-0).

\section{Received: 17 January 2018 Revised: 12 March 2018 Accepted: 14 March} 2018

Published online: 30 April 2018

\section{References}

1. Holohan, C., Van Schaeybroeck, S., Longley, D. B. \& Johnston, P. G. Cancer drug resistance: an evolving paradigm. Nat. Rev. Cancer 13, 714 (2013).

2. Wu, Q., Yang, Z., Nie, Y., Shi, Y. \& Fan, D. Multi-drug resistance in cancer chemotherapeutics: Mechanisms and lab approaches. Cancer Lett. 347, 159-166 (2014).

3. Zhao, Y., Butler, E. B. \& Tan, M. Targeting cellular metabolism to improve cancer therapeutics. Cell Death Dis. 4, e532 (2013).

4. Cantor, J. R. \& Sabatini, D. M. Cancer cell metabolism: one hallmark, many faces. Cancer Discov. 2, 881-898 (2012).

5. Liu, X. S., Little, J. B. \& Yuan, Z. Glycolytic metabolism influences global chromatin structure. Oncotarget 6, 4214-4225 (2015).

6. Efimova, E. V. et al. Linking cancer metabolism to DNA repair and accelerated senescence. Mol. Cancer Res. 14, 173-184 (2016).

7. Porporato, P., Dhup, S., Dadhich, R. K., Copetti, T. \& Sonveaux, P. Anticancer targets in the glycolytic metabolism of tumors: a comprehensive review. Front. Pharmacol. 2, 49-49 (2011).

8. Cairns, R. A., Harris, I. S. \& Mak, T. W. Regulation of cancer cell metabolism. Nat. Rev. Cancer 11, 85 (2011).

9. Palorini, R. et al. Glucose starvation induces cell death in K-ras-transformed cells by interfering with the hexosamine biosynthesis pathway and activating the unfolded protein response. Cell Death Dis. 4, 1-14 (2013).
10. Slawson, C., Copeland, R. J. \& Hart, G. W. O-GlcNAc signaling: a metabolic link between diabetes and cancer? Trends Biochem. Sci. 35, 547-555 (2010).

11. Zachara, N. E., Molina, H., Wong, K. Y., Pandey, A. \& Hart, G. W. The dynamic stress-induced "O-Glcnac-ome" highlights functions for O-GICNAc in regulating DNA damage/repair and other cellular pathways. Amino Acids 40, 793-808 (2011).

12. Butkinaree, C., Park, K. \& Hart, G. W. O-Linked $\beta$-N-acetylglucosamine (OGlcNAc): extensive crosstalk with phosphorylation to regulate signaling and transcription in response to nutrients and stress. Biochim. Biophys. Acta 1800, 96-106 (2010).

13. Zhong, J. et al. Quantitative phosphoproteomics reveals crosstalk between phosphorylation and O-GlcNAc in the DNA damage response pathway. Proteomics 15, 591-607 (2015).

14. Miura, Y., Sakurai, Y. \& Endo, T. O-GlcNAc modification affects the ATMmediated DNA damage response. Biochim. Biophys. Acta 1820, 1678-1685 (2012).

15. Yang, W. H. et al. Modification of p53 with O-linked N-acetylglucosamine regulates p53 activity and stability. Nat. Cell Biol. 8, 1074-1083 (2006).

16. Hamiel, C., Pinto, S., Hau, A. \& Wischmeyer, P. E. Glutamine enhances heat shock protein 70 expression via increased hexosamine biosynthetic pathway activity. Am. J. Physiol. Cell. Physiol. 297, C1509-19 (2009).

17. Bolanos, J. P., Almeida, A. \& Moncada, S. Glycolysis: a bioenergetic or a survival pathway? Trends Biochem. Sci. 35, 145-149 (2010).

18. Gao, Y., Miyazaki, J. \& Hart, G. W. The transcription factor PDX-1 is posttranslationally modified by O-linked $\mathrm{N}$-acetylglucosamine and this modification is correlated with its DNA binding activity and insulin secretion in min6 $\beta$ cells. Arch. Biochem. Biophys. 415, 155-163 (2003).

19. Cunha, D. A. D. et al. JunB protects $\beta$-cells from lipotoxicity via the XBP1-AKT pathway. Cell Death Differ. 21, 1313-1324 (2014).

20. Wang, Z. V. et al. Spliced X-box binding protein 1 couples the unfolded protein response to hexosamine biosynthetic pathway. Cell 156, 1179-1192 (2014).

21. Denisova, O. V. et al. Akt inhibitor MK2206 prevents influenza pH1N1 virus infection in vitro. Antimicrob. Agents Chemother. 58, 3689-3696 (2014).

22. Matsuo, J. et al. Preventing the unfolded protein response via aberrant activation of 4E-binding protein 1 by versipelostatin. Cancer Sci. 100, 327-333 (2009).

23. Chuh, K. N. et al. The new chemical reporter 6-alkynyl-6-deoxy-GlcNAC reveals O-GICNAc modification of the apoptotic caspases that can block the cleavage/activation of caspase-8. J. Am. Chem. Soc. 139, 7872-7885 (2017).

24. Ortizmeoz, R. F. et al. A small molecule that inhibits OGT activity in cells. ACS Chem. Biol. 10, 1392-1397 (2015).

25. Singh, J. P., Zhang, K, Wu, J. \& Yang, X. O-GlcNAc signaling in cancer metabolism and epigenetics. Cancer Lett. 356, 244-250 (2015).

26. Ma, Z. \& Vosseller, K. Cancer metabolism and elevated O-GIcNAc in oncogenic signaling. J. Cell Biol. 289, 34457-34465 (2014).

27. Wang, P. et al. OGT mediated histone H2B S112 GlcNAcylation regulates DNA damage response. J. Genet. Genom. 42, 467-475 (2015).

28. Wang, Z., Huang, Y. \& Zhang, J. Molecularly targeting the PI3K-Akt-mTOR pathway can sensitize cancer cells to radiotherapy and chemotherapy. Cell Mol. Biol. Lett. 19, 233-242 (2014).

29. Guo, Y. et al. MiR-34a inhibits lymphatic metastasis potential of mouse hepatoma cells. Mol. Cell Biochem. 354, 275-282 (2011). 